\title{
Levo-tetrahydropalmatine inhibits the acquisition of ketamine-induced conditioned place preference by regulating the expression of ERK and CREB phosphorylation in rats
}

\author{
Yan $\mathrm{Du}^{\mathrm{ac}}, \mathrm{Li} \mathrm{Du}^{\mathrm{b}}, \mathrm{Jie}_{\mathrm{Cao}}^{\mathrm{c}}$, Christian Hölscher ${ }^{\mathrm{d}, \mathrm{e}}$, Yongming Feng ${ }^{\mathrm{c}}$, Hongliang $\mathrm{Su}^{\mathrm{c}}$, \\ Yujin Wang ${ }^{\mathrm{c}}$, Ke-Ming Yun ${ }^{\mathrm{c}}$ \\ a School of Pharmaceutical Science, Shanxi Medical University, No 56, Xinjian Nan Road, \\ Taiyuan, 030001, Shanxi, China \\ ${ }^{\mathrm{b}}$ The Tumor Hospital of Shanxi Province, Taiyuan, Shanxi, China \\ ' School of Forensic Medicine, Shanxi Medical University, No 56, Xinjian Nan Road, Taiyuan, \\ 030001, Shanxi, China \\ ${ }^{\mathrm{d}}$ Biomedical and Life Science, Faculty of Health and Medicine, Lancaster University, Lancaster \\ LAI 4YQ, UK \\ e Second Hospital, Shanxi Medical University, Taiyuan, Shanxi, PR China
}

\section{accepted in Behav. Brain Research}

Corresponding author:

Dr. Yujin Wang

School of Forensic Medicine, Shanxi Medical University, No 56 Xinjian Nan Road, Taiyuan, 030001, Shanxi, China E-mail address: cxddy_2004@sxmu.edu.cn (Y-J. Wang)

Abbreviations: l-THP, levo-tetrahydropalmatine; ERK, extracellular-regulated kinase; CREB, cAMP responsive element binding protein; Hip, hippocampus; $\mathrm{CPu}$, caudate putamen; $\mathrm{PFc}$, prefrontal cortex; $\mathrm{CPP}$, conditioned place preference; PCP, phencyclidine; NMDA, N-methyl-D-aspartate. 


\section{Abstract:}

Levo-tetrahydropalmatine (l-THP) is an alkaloid purified from the Chinese herbs Corydalis and Stephania and has been used in many traditional Chinese herbal preparations for its sedative, analgesic and hypnotic properties. Previous studies demonstrated that $l$-THP has antagonistic activity on dopamine receptors; thus, it may have potential therapeutic effects on drug abuse. However, whether $l$-THP affects ketamine-induced conditioned place preference (CPP) remains unclear. Therefore, the present study was designed to evaluate the effects of $l$-THP on the rewarding behavior of ketamine through CPP. Results revealed that ketamine $(5,10$ and $15 \mathrm{mg} / \mathrm{kg}$ ) induced CPP in rats. Furthermore, Ketamine $(10 \mathrm{mg} / \mathrm{kg})$ promoted the phosphorylation of extracellular-regulated kinase (ERK) and cAMP responsive element binding protein (CREB) in the hippocampus (Hip) and caudate putamen $(\mathrm{CPu})$, but not in the prefrontal cortex $(\mathrm{PFc}) . l$-THP $(20 \mathrm{mg} / \mathrm{kg})$ co-administered with ketamine during conditioning inhibited the acquisition of ketamine-induced CPP in rats. Furthermore, $l$-THP $(20 \mathrm{mg} / \mathrm{kg})$ prevented the enhanced phosphorylation of ERK and CREB in $\mathrm{CPu}$ and Hip. These results suggest that $l$-THP has potential therapeutic effects on ketamine-induced CPP. The underlying molecular mechanism may be related to its inhibitory effect on ERK and CREB phosphorylation in Hip and $\mathrm{CPu}$. The present data supports the potential use of $l$-THP for the treatment of ketamine addiction.

Keywords: Levo-tetrahydropalmatine; ketamine; conditioned place preference; extracellular regulated protein kinase; cAMP responsive element binding protein 


\section{Introduction}

Ketamine is commonly used in surgery for its anesthetic, sedative, and analgesic properties [1]. Over the past decade, ketamine has emerged as a recreational drug [2], and the abuse of ketamine is increasing year by year. Long-term use of ketamine produced persistent changes in behavior and in brain structure associated with learning and memory [3]. Memories of the learned association between cues and the rewarding properties of abused drugs are difficult to extinguish and this contributes significantly to the high propensity to relapse [4]. Previous studies demonstrated that the mesolimbic dopamine system significantly affects drug-induced behavioral and neuronal changes [5]. The neural circuitry of drug-seeking behavior involves the prefrontal cortex $(\mathrm{PFc})$, caudate putamen $(\mathrm{CPu})$ and the nucleus accumbens $(\mathrm{NAc})[6]$. Ketamine can improve the release of dopamine in the NAc and activate dopamine $\mathrm{D}_{1}$ receptors in the PFc by reducing glutamatergic inhibition of dopaminergic transmission and reducing dopaminergic re-uptake $[7,8]$. Ketamine is similar to other addictive drugs in terms of affecting behavior, such as locomotor sensitization [8,9], self-administration [10] and conditioned place preference (CPP) [11], which is a standard test of the rewarding/addictive properties of drugs $[12,13]$. Therefore, the dopaminergic system is increasingly recognized as a therapeutic target for drug addiction yet there is still no medication currently available for treating ketamine addiction in clinical practice.

Tetrahydropalmatine (THP) is a major active ingredient of Corydalis ambigua and Stephania tetranada; it has sedative, neuroleptic, and analgesic effects [14]. Pharmacological research has revealed that $l$-THP is an antagonist of dopamine $\mathrm{D}_{1}$ and $\mathrm{D}_{2}$ receptors; $l$-THP is more closely associated with dopamine $\mathrm{D}_{1}$ receptors than with 
$\mathrm{D}_{2}$ receptors [15]. l-THP also affects dopamine $\mathrm{D}_{3}$ receptors $[16,17]$. These profiles of l-THP suggest that it may have potential therapeutic effects on drug addiction [18]. l-THP can attenuate methamphetamine-induced locomotor sensitization [19], CPP [20], oxycodone-induced CPP [21], and cocaine self-administration [17]. However, the effect of $l$-THP on the rewarding behavior of ketamine in rats and its underlying molecular mechanisms remains unclear.

ERK is a kinase that plays important roles in second messenger signaling pathways and is implicated in playing a role in the development of addiction to various drugs, including cocaine and methamphetamine. The phosphorylation of ERK is enhanced by addictive drugs [22,23], and this process is related to methamphetamine-induced rewarding behavior [24]. Furthermore, the inhibition of ERK phosphorylation attenuates cocaine-induced CPP [25]. CREB regulates gene expression in cell signaling pathways [26] and is also closely related to drug addiction. Morphine enhances CREB phosphorylation in the hippocampus (Hip) of mice exhibiting CPP [27]. Thus, ERK and CREB phosphorylation may be significantly related to drug-abuse properties. Nevertheless, studies have yet to clarify the mechanism by which $l$-THP modulates the development of ketamine-induced CPP and the relevance of the activation of ERK and CREB phosphorylation in the brain of rats.

In the present study, a CPP model was used to determine the effects of $l$-THP on the rewarding behavior of ketamine. The relationship between rewarding behaviors and changes in ERK and CREB phosphorylation in CPu, Hip and PFc in Sprague-Dawley (SD) rats was investigated. 


\section{Materials \& Methods}

\subsection{Animals}

Male SD rats (200-220 g) were purchased from the Academy of Military Medical Sciences (AMMS, China). All the animals were maintained on $12 \mathrm{~h} / 12 \mathrm{~h}$ light/dark cycle at $22 \pm 2{ }^{\circ} \mathrm{C}$ and $55 \pm 5 \%$ humidity with food and water ad libitum, and were acclimatized for 1 week. The rats were handled daily for 1 week to adapt to the situation before treatment. All experimental procedures involving animals were approved by the Institutional Animal Care and Use Committee of Shanxi Medical University and accordance with the guidelines of National Institute of Health (NIH) guideline (NIH publication NO. 8023. Revised 1978).

\subsection{Reagents}

$l$-THP (99.00\%) was purchased from the Sigma-Aldrich Inc. (USA) and it was dissolved in distilled water. Ketamine hydrochloride was obtained from Hengrui pharmaceutical factory (Jiang Xi, RP China). Rabbit anti-P-ERK antibody and rabbit anti-ERK antibody were acquired from Cell Signaling Technology (USA). Goat anti-rabbit IgG antibody was purchased from Boster Company (Wuhan, RP China). Rabbit anti-CREB and rabbit anti-P-CREB antibody were procured from Abcam (Cambridge, UK). The volume of intraperitoneal (i.p.) injection was $10.0 \mathrm{ml} / \mathrm{kg}$.

\subsection{Experimental design}




\subsubsection{Ketamine addiction experiment.}

In this experiment, different doses of ketamine were administered to reveal the rewarding effect of ketamine. The rats were divided into four groups randomly ( $\mathrm{n}=7$ per group) consisting of (i) a control group treated with saline alone; (ii) a group treated with ketamine of $5 \mathrm{mg} / \mathrm{kg}$; (iii) a group treated with ketamine of $10 \mathrm{mg} / \mathrm{kg}$; (iv) a group treated with ketamine of $15 \mathrm{mg} / \mathrm{kg}$.

\subsection{2 l-THP therapeutic experiment.}

In this experiment, the dose of ketamine was $10 \mathrm{mg} / \mathrm{kg}$; different doses of $l$-THP were treated in combination to study the therapeutic effect of $l$-THP. The rats were divided into four groups randomly ( $\mathrm{n}=6$ per group) consisting of (a) a control group treated with saline alone; (b) a group treated with ketamine of $10 \mathrm{mg} / \mathrm{kg}$; (c) a group treated with ketamine of $10 \mathrm{mg} / \mathrm{kg}$ in combination with $l$-THP of $10 \mathrm{mg} / \mathrm{kg}$; (d) a group treated with ketamine of $10 \mathrm{mg} / \mathrm{kg}$ in combination with $l$-THP of $20 \mathrm{mg} / \mathrm{kg}$. l-THP was administered 30 min before ketamine was administered.

\subsection{CPP apparatus}

The CPP apparatus (JLBeHv, China) consists of two equal-sized compartments (L $* \mathrm{~W} * \mathrm{H}: 30 \mathrm{~cm} * 30 \mathrm{~cm} * 40 \mathrm{~cm}$ ) with a $7 \mathrm{~cm} * 10 \mathrm{~cm}$ sliding door in the center of the base. One compartment had $2 \mathrm{~cm}$ wide black and white horizontal stripes and a wire-mesh floor, whereas the other compartment had $2 \mathrm{~cm}$ wide black and white 
vertical stripes and a bar metal grid rod floor [28]. The time that the rats spent in each compartment were recorded by infrared monitoring system.

\subsection{Conditioned place preference procedure}

The ketamine-induced CPP was examined by a three-phase CPP procedure similar to that described before [29,30] with modifications (Fig.1A). Briefly, the experiment consisted of pre-test phase (1 day); conditioning training phase (10 days), and post-test phase (1 day). For pre-test phase (the 1 th day), each rat was drug free and was placed in the compartment randomly with free access to the entire CPP apparatus for $15 \mathrm{~min}$. The time of rat spent in each compartment was recorded. The rat who spends over $600 \mathrm{~s}$ of the 15 min in either compartment were excluded from further analysis (10\% of all animals). For conditioning training phase (from the 2 th day to the 11th day), ketamine was paired with the non-preferred side (horizontal stripes compartment). On the 2th day, Group (i) received an i.p. saline injection and was confined in the vertical stripes compartment for $40 \mathrm{~min}$; meanwhile, Groups (ii)-(iv) received different doses of ketamine $(5 \mathrm{mg} / \mathrm{kg}, 10 \mathrm{mg} / \mathrm{kg}$, and $15 \mathrm{mg} / \mathrm{kg}$ ) injection (i.p.) respectively and they were confined in the horizontal stripes compartment for $40 \mathrm{~min}$. All the groups received an i.p. saline injection and were confined in the vertical stripes compartment for 40min on the 3th day. Groups (ii)-(iv) received ketamine and saline injections alternatively in two consecutive days which was referred to as a conditioning session, while Group (i) received i.p. saline in both compartments. All the groups experienced a total of five conditioning sessions from 
the 2th to 11th day. During the post-test phase (the 12th day), all the rats were allowed to explore the two compartments freely for $15 \mathrm{~min}$ without injections. The time the rats spent on the drug-paired side (horizontally striped box) was recorded.

To study the effect of $l$-THP on the acquisition of ketamine-induced CPP, $l$-THP was administered 30 min before ketamine injections during the conditioning phase (the 2th, 4th, 6th, 8th, 10th day) respectively.

\subsection{Sample preparation}

All rats were sacrificed on the 12th day after the behavior test was completed. The whole brain was collected rapidly. The Hip, $\mathrm{PFc}$ and the $\mathrm{CPu}$ were dissected and stored at $-80{ }^{\circ} \mathrm{C}$ for Western blot analysis.

\subsection{Western blot}

Western blot assay was performed using standard protocols. The samples were homogenized in ice-cold RIPA lysis buffer (Beyotime Institute of Biotechnology, Shanghai, China) containing phenyl-methylsulfonyl fluoride (PMSF). Homogenates were incubated in an ice bath for $3 \mathrm{~h}$ and then centrifuged at 12,000 rpm for $20 \mathrm{~min}$ at 4 ${ }^{\circ} \mathrm{C}$. The protein concentration of the samples then was quantified by Bradford BCA protein assay. After tissue lysate was mixed in loading buffer and boiled for $5 \mathrm{~min}$, equal amounts of denatured total protein were loaded and separated through $12 \%$ sodium dodecyl sulfate-polyacrylamide gel electrophoresis. The proteins were transferred to $0.45 \mu \mathrm{m}$ polyvinylidene difluoride (PVDF) membranes. The membranes 
were blocked with $5 \%$ BSA in tris-buffered saline for $2 \mathrm{~h}$ at $4{ }^{\circ} \mathrm{C}$ and then incubated with primary antibodies, namely, anti-p-CREB (1:5000), anti-p-ERK (1:2000), anti-CREB (1:750), and anti-ERK (1:1000) at $4{ }^{\circ} \mathrm{C}$ overnight. The membranes were incubated with the corresponding secondary antibodies at $4{ }^{\circ} \mathrm{C}$ for $2 \mathrm{~h}$. Bands were visualized by using the enhanced chemiluminescence kit according to the manufacturer's instruction. Western blots were scanned and analyzed by Alpha View SA Image software.

\subsection{Data analysis and statistical analysis}

The place preference score was defined as the time spent in the drug-paired chamber during the post-test session minus the time spent in the drug-paired chamber during the pre-test session [31].

SPSS version 16.0 statistical software (SPSS, Inc., Chicago, Il, USA) was used for statistical analysis. One-way ANOVA with post-hoc multiple comparisons (Bonferroni test) was performed to analyze multi-group. Data are presented as mean \pm SEM. A $p$-value $<0.05$ was considered to indicate a statistically significant difference.

\section{Results}

\subsection{Ketamine-induced place preference}

As shown in Fig. 1B, the saline-control rats exhibited no preference for either the drug- or saline-associated compartment. The mean CPP score was $25.7 \pm 9.7$ sec. 
Ketamine $(5-15 \mathrm{mg} / \mathrm{kg}$, i.p. $)$ produced place preference $\left(F(3,24)=5.986,{ }^{*} p<0.05\right)$. The mean conditioning scores were $104.3 \pm 3.5 \mathrm{sec}\left(5 \mathrm{mg} / \mathrm{kg},{ }^{*} p<0.05\right), 132.3 \pm 4.6$ $\sec (10 \mathrm{mg} / \mathrm{kg}, * p<0.05)$ and $153.1 \pm 16.6 \mathrm{sec}(15 \mathrm{mg} / \mathrm{kg}, * p<0.05) . \mathrm{N}=7 \mathrm{per}$ group.

\subsection{Effects of l-THP on the acquisition of ketamine-induced place preference}

As shown in Fig. 1C, Ketamine $(10 \mathrm{mg} / \mathrm{kg}$, i.p. $)$ produced a significant preference for the drug-associated place. The mean conditioning scores was $137.8 \pm$ $6.2 \mathrm{sec}$. However, the effect was inhibited by pretreatment with $l$-THP $(20 \mathrm{mg} / \mathrm{kg})$ : the mean conditioning scores was $38.2 \pm 8.8 \sec (F(3,20)=8.665, \# p<0.05)$. While, l-THP $(10 \mathrm{mg} / \mathrm{kg})$ did not significantly affect the development of ketamine-induced CPP: the mean conditioning scores was $118 \pm 9.7 \sec (p>0.05) . \mathrm{N}=6$ per group.

\subsection{Effect of l-THP on ERK and CREB phosphorylation in Hip from conditioned place} preference rats

As shown in Fig. 2A, ketamine $(10 \mathrm{mg} / \mathrm{kg})$ had significant effects on the level of p-ERK in the Hip (*p $<0.05$ vs. saline group) in rats. The level of p-ERK in $l$-THP $(10 \mathrm{mg} / \mathrm{kg}$ ) pre-treatment group was slight lower than ketamine group, but there was no significance $(p>0.05)$. However, ketamine-induced activation of $\mathrm{p}$-ERK was significantly reversed by pre-treatment with $l$-THP $(20 \mathrm{mg} / \mathrm{kg})$ in the conditioning phase $(F(3,20)=5.150, \# p<0.05$ vs. ketamine group $) . \mathrm{N}=6$ per group. 
As shown in Fig. 2B, the level of p-CREB (in the Hip) in alone ketamine group significantly increased $\left({ }^{*} p<0.05\right)$, compared with saline group. $l$-THP $(10 \mathrm{mg} / \mathrm{kg})$ pre-treatment could not significantly reverse p-CREB in Hip $(p>0.05)$. However, $l$-THP $(20 \mathrm{mg} / \mathrm{kg})$ pre-treatment significantly inhibited the increase of p-CREB's level in $\operatorname{Hip}(F(3,8)=17.90, \# p<0.05$, compared with ketamine group). $\mathrm{N}=3$ per group.

\subsection{Effect of l-THP on ERK and CREB phosphorylation in CPu from conditioned place} preference rats

Compared with saline, ketamine significantly increased the level of p-ERK in the $\mathrm{CPu}\left({ }^{*} p<0.05\right)$. Multiple comparisons revealed that the ketamine-induced activation of p-ERK was significantly prevented by the pre-treatment of $l$-THP (10 and $20 \mathrm{mg} / \mathrm{kg}$ ) in the conditioning phase $(F(3,16)=10.42, \# p<0.05$, compared with ketamine group). $\mathrm{N}=3$ per group. These results were shown in Fig. 3A.

The level of p-CREB was significantly increased in the $\mathrm{CPu}$ of the rats in the ketamine group $(* p<0.05$ compared with the saline group). $l$-THP pre-treatment (20 $\mathrm{mg} / \mathrm{kg})$ significantly reversed the increase $(F(3,8)=5.299, \# p<0.05$, compared with the ketamine group). These results were shown in Fig. 3B. $\mathrm{N}=3$ per group.

\subsection{Effect of l-THP on ERK and CREB phosphorylation in PFc from conditioned place} preference rats

We attempted to find out whether ketamine or $l$-THP increased the level of CREB Ser133 phosphorylation in PFc from conditioned place preference rats. The results 
demonstrated that ketamine or $l$-THP did not alter the level of ERK phosphorylation in PFc from conditioned place preference rats $(F(3,8)=0.4647, p>0.05)$. These results were shown in Fig. 4A. $\mathrm{N}=3$ per group.

As shown in Fig. 4B, neither ketamine nor $l$-THP could significantly activate p-CREB in $\operatorname{PFc}(F(3,8)=0.3657, p>0.05) . \mathrm{N}=3$ per group.

\section{Discussion}

In our study we show for the first time that the drug $l$-THP, a DA receptor antagonist that also affects adrenergic and serotonergic receptors [32,33] inhibits the acquisition of ketamine-induced conditioned place preference (CPP) by regulating the expression of ERK and CREB phosphorylation in the brain of rats. Previous studies demonstrate that $l$-THP can attenuate methamphetamine-induced CPP [20], oxycodone-induced CPP [21], cocaine self-administration [17], and heroin self-administration [34], but no data has been published previously that demonstrates that $l$-THP may also have effects on the rewarding behavior of ketamine. In the present study, ketamine $(5,10$ and $15 \mathrm{mg} / \mathrm{kg}$, i. p.) induced CPP in the rats. This conclusion is consistent with that described in previous studies [35-37]. Besides, we found that $l$-THP $(1.25,2.5,5.0,10.0,20.0 \mathrm{mg} / \mathrm{kg}$, i.p.) alone did not produce conditioned place preference [20]. However, l-THP inhibited the acquisition of conditioned place preference induced by ketamine. These results suggest that $l$-THP may be useful for the treatment of ketamine addiction. 
The conditioned place preference paradigm has been widely used as a model for studying the rewarding effect of drugs with addictive properties. The actual learning process in this task can be manifold, including classical conditioning but also operant conditioning [13]. Hence, one has to exercise caution when assigning specific learning mechanisms and brain areas to the drug effects in the interpretation of the experiments. Many drugs, such as amphetamine, cocaine and opioids can induce CCP [38]. In the present study, we found that ketamine induced CCP in rats. It has been previously shown that ketamine has rewarding effects with addictive properties [39]. Ketamine is a N-methyl-D-aspartate (NMDA) receptor antagonist, and previous studies demonstrated that NMDA receptor antagonists can induce rewarding behavior [35-37]. The drug activates the mesolimbic dopamine reward system by reducing the glutamatergic inhibition on dopaminergic transmission. For instance, several neurochemical studies have revealed that ketamine increases dopamine release in the nucleus accumbens [40]. In addition, NMDA receptor antagonists can increase extracellular dopamine levels by inhibiting dopamine reuptake [35,39].

The mechanism by which $l$-THP inhibits the rewarding effects of ketamine is most likely by blocking dopaminergic receptors in the nucleus accumbens. Dopaminergic transmission drives the reward system, and activation of dopamine receptors is related to cocaine-induced CPP [22]. Moreover, the microinjection of dopamine receptor antagonists into relevant brain regions attenuates the development of addictive behaviors [41]. The pharmacological properties of $l$-THP include the antagonism of dopamine D1 and D2 receptors and the actions at D3 receptors [18]. Therefore, $l$-THP 
may have potential effects on the treatment of drug abuse. Reports showed that $l$-THP can attenuate methamphetamine-induced CPP [20], oxycodone-induced CPP [21], cocaine self-administration [17], and heroin self-administration [34]. In our study, 1-THP was pretreated 30 min before ketamine injection to investigate the effects of 1-THP on adjusting ketamine-induced CPP. We found that 1-THP $(20 \mathrm{mg} / \mathrm{kg})$ could significantly inhibit ketamine-induced CPP in the rat. l-THP attenuated the development of ketamine-induced CPP possibly because they bind to dopamine D1 and D2 receptors and block activation. These results indicate that the inhibiting effect of $l$-THP is due to its antagonistic activity on the dopaminergic system.

Some of the underlying molecular mechanisms based on long-term behavioral changes caused by addictive drugs have been investigated in the present study. The activation of the ERK and CREB pathway in relevant brain regions can induce the development of addictive behaviors. For example, METH- and cocaine-induced CPP occurred when ERK and CREB were activated in distinct brain regions [24]. The pre-injection of the MAPK antagonist abolished the development of cocaine-induced CPP [25]. The activation of ERK induces the direct or indirect phosphorylation of various transcription factors, including CREB, which is essential for long-term behavioral and synaptic changes. Previous studies demonstrated that dopamine D1 receptors are essential for cocaine-induced phosphorylation of ERK in specific brain regions and drug-induced ERK activation can be inhibited by associated dopamine D1 antagonists or D1 receptor mutation [25]. Therefore, although the exact mechanisms remain unclear, these findings indicate that $l$-THP, as a dopamine receptor antagonist, 
may inhibit dopamine receptors in associated brain regions and decrease the level of ERK and CREB phosphorylation, which contribute to the inhibition of ketamine-induced CPP in rats. We therefore analyzed the levels of ERK and CREB phosphorylation in the Hip, $\mathrm{PFc}$ and $\mathrm{CPu}$. There was a significant increase in levels of p-ERK and p-CREB in the ketamine-treated group. Furthermore, $l$-THP $(20 \mathrm{mg} / \mathrm{kg}$ ) pretreatment partially inhibited the increase of p-ERK and p-CREB levels in Hip and $\mathrm{CPu}$. The inhibition of $l$-THP in ketamine-induced CPP performance was consistent with the reduced levels of p-ERK and p-CREB protein levels. The role of $l$-THP on ketamine-induced molecular changes indicated that the inhibition of ERK and CREB phosphorylation in relevant brain regions may be related to the treatment effects of $l$-THP on ketamine-induced rewarding behavior in rats.

Specific dopamine receptor antagonists may be used to alleviate drug addiction because of the effect on the mesolimbic dopamine system in modulating drug abuse [42]. However, dopamine receptor antagonists cannot be used widely because they may potentially reinforce addiction and promote drug abuse [43]. Unlike other dopamine receptor antagonists, $l$-THP derived from traditional Chinese medicine has been used safely for years without inducing side effects, including reinforcing addiction or promoting drug abuse [18]. These results demonstrate that $l$-THP does not impair basic hedonic processes, but rather, specifically inhibits the rewarding efficacy of drugs of abuse. Therefore, $l$-THP could be a potential candidate for the treatment of drug addiction. 


\section{Conclusion}

This study confirmed that $l$-THP can modulate the development of ketamine-induced CPP. Moreover, the inhibition of ERK and CREB phosphorylation in the Hip and CPu may be involved in the effects of $l$-THP on the modulation of ketamine-induced CPP. Therefore, l-THP may be a potential drug treatment for ketamine addiction. However, the precise mechanisms and safe use of $l$-THP for humans should be further investigated.

\section{Acknowledgements}

This research did not receive any specific grants from funding agencies in the public, commercial, or not-for-profit sectors. The authors declare no conflict of interest. 


\section{References}

[1] J. White, C. Ryan, Pharmacological properties of ketamine, Drug Alcohol Rev 15 (1996) 145-155.

[2] Y.X. Fang, Y.B. Wang, J. Shi, Z.M. Liu, L. Lu, Recent trends in drug abuse in China, Acta Pharmacol Sin 27 (2006) 140-144.

[3] K.W. Chan, T.M. lee, A.M. Siu, D.P. Wong, C.M. Kam, S.K. Tsang, Effects of chronic ketamine use on frontal and medial temporal cognition, Addict Behav 38 (2013) 2128-2132.

[4] S.E. Hyman, R.C. Malenka, E.J. Nestler, Nerural mechanisms of addiction: the role of reward-related learning and memory, Annu Rev Neursci 29 (2006) $565-598$.

[5] N.D. Volkow, J.S. Flowler, G.J. Wang, R. Baler, F. Telang, Imaging dopamine's role in drug abuse and addiction, Neuropharmacology 56 (2009) 3-8.

[6] R.M. Carelli, S.G. Ljames, Selective activation of accumbens neurons by cocaine-associated stimuli during a water/cocaine multiple schedule, Brain Res 907 (2001) 156-161.

[7] M. Masuzawa, S. Nakao, E. Miyamoto, M. Yamada, K. Murao, K. Nishi, K. Shingu, Pentobarbital inhibits ketamine-induced dopamine release in the rat nucleus accumbens:a microdialysis study, Anesth Analg 96 (2003) 148-152. 
[8] K.A. Trujillo, J.J. Zamora, K.P. Warmoth, Increased response to ketamine following treatment at long intervals: implications for intermittent use, Biol Psychiatry 63 (2008) 178-183.

[9] J.L. Wiley, R.L. Evans, D.B. Grainger, K.L. Nicholson, Age-dependent differences in sensitivity and sensitization to cannabinoids and 'club drugs' in male adolescent and adult rats, Addict Biol 13 (2008) 277-286.

[10] K.L. Marquis, M.G. Webb, J.E. Moreton, Effects of fixed ratio size and dose on phencyclidine self-administration by rats, Psychopharmacology (Berl) 97 (1989) 179-182.

[11] T. Suzuki, H. Kato, T. Aoki, M. Tsuda, M. Narita, M. Misawa, Effects of the non-competitive NMDA receptor antagonist ketamine on morphine-induced place preference in mice, Life Sci 67 (2000) 383-389.

[12] T.M. Tzschentke, Measuring reward with the conditioned place preference (CPP) paradigm: update of the last decade, Addict Biol 12 (2007) 227-462.

[13] J.P. Huston, M.A. de.Souza.Silva, B. Topic, C.P. Müller, What's conditioned in conditioned place preference?, Trends in Pharmacological Sciences 34 (2013) 162-166.

[14] G.S. Ding, Important Chinese herbal remedies, Clin. Ther 9 (1987) 345-357.

[15] H. Chu, G. Jin, E. Friendman, X. Zhen, Recent development in studies of tetrahydroprotoberberines: mechanism in antinociception and drug addiction, Cell Mol Neurobiol 28 (2008) 491-499. 
[16] J.R. Mantsch, S.J. Li, R. Risinger, S. Awad, E. Katz, D.A. Baker, Z. Yang, Levo-tetrahydropalmatine attenuates cocaine self-administration and cocaine-induced reinstatement in rats, Psychopharmacology(Berl) 192 (2007) $581-591$.

[17] J.R. Mantsch, S. Wisniewski, O. Vranjkovic, C. Peters, A. Becker, A. Valentine, S.J. Li, D.A. Baker, Z. Yang, Levo-tetrahydropalmatine attenuates cocaine self-administration under a progressive-ratio schedule and cocaine discrimination in rats., Pharmacol Biochem Behav 97 (2010) 310-316.

[18] J.B. Wang, J.R. Mantsch, l-tetrahydropalamatine: a potential new medication for the treatment of cocaine addiction, Future Med Chem 4 (2012) 177-186.

[19] N. Zhao, Y. Chen, J. Zhu, L. Wang, G. Cao, Y. Dang, C. Yan, J. Wang, T. Chen, Levo-tetrhydropalmatine attenuates the development and expression of methamphetamine-induced locomotor sensitization and the accompanying activation of ERK in the nucleus accumbens and caudate putamen in mice, Neuroscience 258 (2014) 101-110.

[20] H.L. Su, J. Zhu, Y.J. Chen, N. Zhao, W. Han, Y.H. Dang, M. Xu, T. Chen, Roles of levo-tetrahydropalmatine in modulating methamphetamine reward behavior, Physiol Behav 118 (2013) 195-200.

[21] Y.L. Liu, L.D. Yan, P.L. Zhou, C.F. Wu, Z.H. Gong, Levo-tetrahydropalmatine attenuates oxycodone-induced conditioned place preference in rats, Eur J Pharmacol 602 (2009) 321-327. 
[22] L. Chen, M. Xu, Dopamine D1 and D3 receptors are differentially involved in cue-elicited cocaine seeking, J Neurochem 114 (2010) 530-541.

[23] L. Lu, B.T. Hope, J. Dempsey, S.Y. Liu, J.M. Bossert, Y. Shaham, Central amygdala ERK signaling pathway is critical to incubation of cocaine craving, Nat Neurosci 8 (2005) 212-219.

[24] H. Mizoguchi, K. Yamada, M. Mizuno, T. Mizuno, A. Nitta, Y. Noda, T. Nabeshima, Regulations of methamphetamine reward by extracellular signal-regulated kinase 1/2/ets-like gene-1 signaling pathway via the activation of dopamine receptors, Mol Pharmacol 65 (2004) 1293-1301.

[25] E. Valjent, J.C. Corvol, C. Pages, M.J. Besson, R. Maldonado, J. Caboche, Involvement of the extracellular signal-regulated kinase cascade for cocaine-rewarding properties, J Neurosci 20 (2000) 8701-8709.

[26] F.H. Cruzalegui, E. Cano, R. Treisman, ERK activation induces phosphorylation of Elk-1 at multiple S/T-P motifs to high stoichiometry, Oncogene 18 (1999) 7948-7957.

[27] C. Gao, L. Chen, Y. Tao, J. Chen, X. Xu, G. Zhang, Z. Chi, Colocalization of phosphorylated CREB with calcium/calmodulin-dependent protein kinase IV in hippocampal neurons induced by ohmfentanyl stereoisomers, Brain Res 1024 (2004) 25-33.

[28] M. Moaddab, B.I. Hyland, C.H. Brown, Oxytocin enhances the expression of morphine-induced conditioned place preference in rats, Psychoneuroendocrinology 53 (2015) 159-169. 
[29] R. Hu, R. Song, R. Yang, R. Su, J. Li, The dopamine D(3) receptor antagonist YQA14 that inhibits the expression and drug-prime reactivation of morphine-induced conditioned place preference in rats, Eur J Pharmacol 720 (2013) 212-217.

[30] T. Suzuki, Y. Masukawa, M. Misawa, Drug interactions in the reinforcing effects of over-the-counter cough syrups, Psychopharmacology 102 (1990) $438-442$.

[31] F.Q. Li, Q. Fang, Y. Liu, M. Zhao, D.P. Li, J.S. Wang, Cannabinoid CB1 receptor antagonist rimonabant attenuates reinstatement of ketamine conditioned place preference in rats, Eur J Pharmacol 589 (2008) 122-126.

[32] Z.Z. Lu, X. Wei, G.Z. Jin, Q.D. Han, Antagonistic effect of tetrahydroproberberine homologues on alpha 1-adrenoceptor., Yao Xue Xue Bao 31 (1996) 652-656.

[33] X. Liu, Z. Yang, R. Li, J. Xie, Q. Yin, A.S. Bloom, Responses of dopaminergic, serotonergic and noradrenergic networks to acute levo-tetrahydropalmatine administration in naive rats detected at 9.4 T. , Magn Reson Imaging 30 (2012) 261-270.

[34] K. Yue, B. Ma, Q. Ru, L. Chen, Y. Gan, D. Wang, G. Jin, C. Li, The dopamine receptor antagonist levo-tetrahydropalmatine attenuates heroin self-administration and heroin-induced reinstatement in rats, Pharmacol Biochem Behav 102 (2012) 1-5. 
[35] H. Morris, J. Wallach, From PCP to MXE: a comprehensive review of the non-medical use of dissociative drugs, Drug Test Anal 6 (2014) 614-632.

[36] M.M. Marcus, J.M. Mathe, G.G. Nomikos, T.H. Svensson, Effects of competitive and non-competitive NMDA receptor antagonists on dopamine output in the shell and core subdivisions of the nucleus accumbens, Neuropharmacology 40 (2001) 482-490.

[37] D.D. Xu, Z.X. Mo, K.K. Yung, Y. Yang, A.W. Leung, Individual and combined effects of methamphetamine and ketamine on conditioned place preference and NR1 receptor phosphorylation in rats, Neurosignals 15 (2006-2007) 322-331.

[38] M.T. Bardo, J.K. Rowlett, M.J. Harris, CPP using opiate and stimulant drugs: a meta-anaysis., Neurosci Biobehav Rev 19 (1995) 39-51.

[39] C.J. Morgan, H.V. Curran, Ketamine use: a review, Addiction 107 (2012) 27-38.

[40] M. Irifune, T. Fukuda, M. Nomoto, T. Sato, Y. Kamata, T. Nishikawa, W. Mietani, K. Yokoyama, K. Sugiyama, M. Kawahara, Effects of ketamine on dopamine metabolism during anesthesia in discrete brain regions in mice: comparison with the effects during the recovery and subanesthetic phases, Brain Res 763 (1997) 281-284.

[41] Z.X. Xi, E.A. Stein, GABAergic mechanisms of opiate reinforcement, Alcohol Alcohol 37 (2002) 485-494. 
[42] D. Quarta, I. Smolders, Rewarding, reinforcing and incentive salient events involve orexigenic hypothalamic neuropeptides regulating mesolimbic dopaminergic neurotransmission, Eur J Pharm Sci 57 (2014) 2-10.

[43] M.R. Weed, I.A. Paul, L.P. Dwoskin, S.E. Moore, W.L. Woolverton, The relationship between reinforcing effects and in vitro effects of D1 agonists in monkeys, J Pharmacol Exp Ther 283 (1997) 29-38. 


\section{Figure captions}

Fig. 1 (A) The conditioned place preference (CPP) behavioral schedule. K: ketamine, S: saline. (B) Effect of different doses of ketamine on place conditioning. Data are expressed as mean \pm SEM. $\left(\mathrm{n}=7\right.$ per group). ${ }^{*} \mathrm{p}<0.05$ compared with the saline group; (C) Effect of pretreatment with 1-THP (10, $20 \mathrm{mg} / \mathrm{kg}$, i.p.) on the place conditioning produced by ketamine $(10 \mathrm{mg} / \mathrm{kg}$, i.p. $)$ in rats. Rats were pretreated with 1-THP $30 \mathrm{~min}$ prior to each ketamine injection. Data are expressed as mean \pm SEM. ( $n=6$ per group). $* \mathrm{p}<0.05$ vs. saline group, \# $\mathrm{p}<0.05$ vs. the ketamine group.

Fig. 2 (A) Effect of 1-THP on p-ERK in the Hip from conditioned place preference rats;

(B) Effect of 1-THP on p-CREB in the Hip from conditioned place preference rats. Values are expressed as mean $\pm \mathrm{SEM}, * \mathrm{p}<0.05$ vs. saline group, $\# \mathrm{p}<0.05$ vs. the ketamine group.

Fig. 3 (A) Effect of 1-THP on p-ERK in the CPu from conditioned place preference rats;

(B) Effect of 1-THP on $\mathrm{p}-\mathrm{CREB}$ in the $\mathrm{CPu}$ from conditioned place preference rats. Values are expressed as mean \pm SEM. $* \mathrm{p}<0.05$, compared with the saline group, $\# \mathrm{p}<$ 0.05, compared with the ketamine group.

Fig. 4 (A) Effect of 1-THP on p-ERK in PFc from conditioned place preference rats; (B) Effect of 1-THP on p-CREB in PFc from conditioned place preference rats. Values are 
expressed as mean \pm SEM. $* \mathrm{p}<0.05$, compared with the saline group, \# $\mathrm{p}<0.05$, compared with the ketamine group. 